\title{
MODELLING ADOLESCENT PEDESTRIAN CROSSING DECISION AT UNMARKED ROADWAY
}

\author{
PENG CHEN, JINGMIN XIE \& JINGLIU YU \\ School of Transportation, Wuhan University of Technology, China.
}

\begin{abstract}
To address adolescent pedestrian safety problems at unmarked roadway, there is a need to understand adolescent pedestrian crossing decision behavior at unmarked roadway. Cloud model, which is an uncertainty conversion model between qualitative knowledge description and quantitative value expression, was used to deal with adolescent pedestrian's cognitive uncertainty in the crossing decisionmaking process. Then the decision table for adolescent pedestrian crossing at unmarked roadway was established. Attribute reduction based on discernibility matrix and value reduction based on induction in the rough set theory were applied to reduce the decision table and extract the decision rules of adolescent pedestrian crossing at unmarked roadway. After the reduction, the conditional attributes were the vehicle speed and the distance between pedestrian and vehicle. Nine crossing decision rules were obtained, including five certainty rules. Finally, the proposed method was compared with the existing method. The results show that the prediction accuracy and area under the receiver operating characteristic (ROC) curve for the proposed method are $91.4 \%$ and 0.941 respectively, so it is superior to the Logistic regression model, and the simple and intuitive pedestrian crossing decision rules can be obtained, which can lay the foundation for traffic safety simulation.
\end{abstract}

Keywords: adolescent pedestrian, cloud model, crossing decision, gap acceptance, rough set, unmarked roadway.

\section{INTRODUCTION}

Adolescent pedestrians are a major population at risk of being killed or injured in traffic accidents in the world. Many of traffic accidents occurred when adolescent pedestrians were crossing the roads [1]. To address adolescent pedestrian safety problem at unmarked roadway, there is a need to better understand adolescent pedestrian crossing decision behavior at unmarked roadway.

Over the years, extensive research has been conducted on modelling pedestrian crossing behavior. Some work has been dedicated to identifying significant factors that affect crossing behavior. Zhuang and $\mathrm{Wu}$ [2] explored pedestrian crossing behavior at unmarked roadway in China. It was found that vehicle type and group size are the important predictors for safety margin and waiting time does not have significant linear relationship with safety margin. Xie et al. [3] investigated pedestrian jaywalking at signalized crosswalks. The gender and walking speed of the pedestrian, vehicle flow, and site location and condition of the crosswalk were found to significantly determine the probability of pedestrian jaywalking.

Meanwhile, researchers attempted to develop gap acceptance models to predict the likelihood of pedestrian crossing. Using the probit and binary logit approach, Sun et al. [4] established models to estimate the probability of pedestrian gap acceptance. The model showed that gap size, number of waiting pedestrians, and pedestrian age were the vital attributes for pedestrian crossing behaviors. Yannis et al. [5] investigated pedestrians' traffic gap acceptance for mid-block street crossing in urban areas. A lognormal regression model was developed to examine pedestrian gap acceptance. A binary logistic regression model was developed to examine the effect of traffic gaps and other parameters on pedestrians' decisions to cross the street or not. Their research concluded that pedestrian speed, vehicle speed, 
crossing direction, gap size and pedestrian age all influenced the decision-making of pedestrian crossing. Mamidipalli et al. [6] proposed probit-based pedestrian gap acceptance model for midblock crossing locations. Their model involved only two parameters: the size of the gap length in seconds and a binary variable that distinguished between gaps and lag events. Liu and Tung [7] constructed logistic regression model to investigate the effects of age, time gap, time of day, and speed of approaching vehicle on the decision of pedestrians to cross a road. Their study found that pedestrians' decisions on whether or not they would cross the road safely were made based on the distance between them and the oncoming vehicle. Pawar and Patil [8] explored pedestrian temporal and spatial gap acceptance at uncontrolled midblock street crossings. Their paper reported the applicability and generality of driver's gap acceptance models to the pedestrian's gap acceptance and found that speed of the conflicting vehicle has significant effect on the spatial gap acceptance.

These studies mainly adopted probit or logit approach to describe the relationship between explanatory variables and the probability of outcome of crossing decision-making, but it needs to create the complex function and could not extract the certainty rules. A recent research conducted by Papadimitriou et al. [9] analyzed road, traffic, and human factors of pedestrian crossing behavior through the development of integrated choice and latent variables models. However, the approach is also a computationally demanding technique. In addition, the models mentioned above could not reflect the cognitive fuzziness and randomness for influencing factors in pedestrian crossing decision.

This paper adopted the cloud model $[10,11]$ to describe the cognitive uncertainty for influencing factors in pedestrian crossing decision and discretize the continuous attribute data. Then adolescent pedestrian crossing decision table was constructed and the rough set method $[12,13]$ was used to extract crossing decision rules expressed in the form of if-then statements, which make knowledge have a clear meaning.

\section{PRELIMINARY}

\subsection{Cloud theory}

Cloud model $[10,11]$ can describe the randomness and fuzziness of concepts and make conversion between qualitative concepts and quantitative values. Digital characteristics of cloud model can reflect the overall properties of the concept.

The Cloud is defined as follows: assuming that $U$ is a quantitative domain expressed by exact numerical, $C$ is a qualitative concept on the domain, $x$ is a quantitative value, $x \in U$ and $x$ is stochastic realization of $C, u(x)$ represents the certainty degree of $x$ belonging to concept $C, u(\mathrm{x}) \in[0,1]$ is a random variable with stable tendency. If $u: U \rightarrow[0,1] \forall x \in U x \rightarrow u(\mathrm{x})$, then, we call the distribution of $x$ on domain cloud, $x$ is called as a cloud drop. In the cloud model, the digital characteristics of cloud model are denoted as Expected value Ex, Entropy $E n$ and Hyper entropy $\mathrm{He}$, and they reflect the whole characteristics of the quality conception $C$. Ex is expected value which comes from the distribution of cloud drops samples on domain space; $E n$ is a criterion of measuring the uncertainty of qualitative concept, it can reflect the randomness and fuzziness of qualitative concept; $\mathrm{He}$ is the second-order entropy of the entropy and co-determined by both the randomness and fuzziness of entropy.

Cloud model uses forward cloud generator and backward cloud generator to transform the qualitative concept and quantitative data. The forward cloud generator transforms qualitative concept to quantitative data. The backward cloud generator is a conversion model, which converts quantitative data to quantitative concept. 


\subsection{Rough set theory}

Rough set theory $[12,13]$ uses information systems to represent knowledge and deal with vague data. Some basic rough set concepts are as follows.

Let $S=(U, A)$ be an information system, where $U$, called universe, is a nonempty set of finite objects; $A$ is a nonempty finite set of attributes such that $a$ : $U \rightarrow V_{a}$ for every $a \in A ; V_{a}$ is the value set of $a$. In a decision system with a set of decision attributes, $A=C \cup D$ where $C$ is the set of condition attributes and $D$ is the set of decision attributes. Such an information system also is called a decision table.

For an attribute set $P \subseteq A$, there is an associated indiscernibility relation $\operatorname{IND}(P)$ : $\operatorname{IND}(P)=\left\{(x, y) \in U^{2} \mid \forall a \in P, a(x)=a(y)\right\}$.

The partition of $U$, generated by $\operatorname{IND}(P)$ is denoted $U / P$. If $(x, y) \in \operatorname{IND}(P)$, then $x$ and $y$ are indiscernible by attributes from $P$. The equivalence classes of the $P$-indiscernibility relation are denoted $[x]_{P}$. The indiscernibility relation is the mathematical basis of rough set theory.

Another fundamental concept is attribute reduction. If one considers a non-empty attributes subset, $R \subset P$ and $I N D(R)=I N D(P)$, then $P-R$ is dispensable. Any minimal $R$ such that $I N D(R)=I N D(P)$, is a minimal set of attributes that preserves the indiscernibility relation computed on the set of attributes $P$. $R$ is called reduct of $P$ and denoted as $R \in R E D(P)$. The core of $P$ is the intersection of these reductions, defined as $\operatorname{CORE}(P)=\bigcap \operatorname{RED}(P)$. Naturally, the core contains all the attributes from $P$ which are considered of greatest importance for classification.

The process by which the maximum number of condition attribute values is removed without losing essential information is called value reduction. After value reduction, rules can be generated by associating the condition attribute values with the corresponding decision class value. Let $S=(U, C \cup D)$ be a decision table, $X_{i} \in U\left|C, Y_{j} \in U\right| D$ and $X_{i} \cap Y_{j} \neq \varnothing$. By $\operatorname{des}\left(X_{i}\right)$ and $\operatorname{des}\left(Y_{j}\right)$, we denote the descriptions of the equivalence classes $X_{i}$ and $Y_{j}$ in the decision table $S$. A decision rule is formally defined as $Z_{i j}: \operatorname{des}\left(X_{i}\right) \rightarrow \operatorname{des}\left(Y_{j}\right)$. The confidence of decision rule $Z_{i j}$ is defined as: $\operatorname{Con}\left(Z_{i j}\right)=\left|X_{i} \cap Y_{j}\right| /\left|X_{i}\right|$. For a certain rule, $\operatorname{Con}\left(Z_{i j}\right)=1$; whereas an uncertain rule, $0<\operatorname{Con}\left(Z_{i j}\right)<1$.

\section{METHOD}

The decision table for adolescent pedestrian crossing can be established by taking influencing factors for pedestrian crossing decision as the condition attribute set and taking pedestrian crossing decision as the decision attribute set. Then the decision rule can be extracted by rough set theory. The following describes adolescent pedestrian crossing decision table discretization method and adolescent pedestrian crossing decision rule extraction method.

\subsection{Crossing decision table discretization}

With reference to the relevant studies, the following influencing factors for adolescent pedestrian crossing decision at unmarked roadway are taken into account: the distance between the vehicle and the pedestrian, vehicle speed, vehicle type and group size. When constructing the decision table, it is necessary to discretize the continuous data of distance and speed. The following describes the discretization processing method for the continuous data of distance and speed. 
When judging the distance and speed, the pedestrian has a certain degree of fuzziness and randomness. It is mainly reflected in the fact that the distance and speed judgment results can be described by natural language (for example, distance is described by far and near, and the speed is described by fast and slow), and the concept in the natural language is qualitative; the subjective differences in pedestrians will lead to the randomness of the same value belonging to a certain of concept. The cloud model portrays the randomness and fuzziness as well as their relationships in linguistic terms and can establish transformation of uncertainty between the qualitative concept and the quantitative description, so it can be used to transform influencing factors for pedestrian crossing decision into qualitative concept and obtain discretized data.

In this paper, the backward cloud generator $[14,15]$ is used to extract the digital characteristics of different concepts for distance and speed from the data sequence of the influencing factors, and then the condition cloud generator is used to calculate the certainty degrees of the given data belonging to the different concepts, and finally the concept corresponding to the given data can be determined by comparing the certainty degrees. The specific process is as follows:

Step 1: Construct the data sequence for different levels of the influencing factors

Firstly, the speed is divided into three levels, that is, fast, medium and slow, and the distance is also divided into three levels, that is, far, medium and near. Then, adolescent pedestrians are invited to judge the level of speed and distance in the pedestrian-vehicle conflict video according to their own perceptions, and the corresponding quantitative data for speed and distance are extracted from the pedestrian-vehicle conflict video. Finally, the quantitative data corresponding to a certain level of a certain influencing factor (speed or distance) are arranged in ascending order to construct the data sequence. The data sequence for a certain level of a certain influencing factor can be recorded as follows: $x=\left[x_{1}, x_{2}, \cdots, x_{n}\right]$

Step 2: Extract the digital characteristics for different levels of the influencing factors

The backward cloud generator is used for digital characteristics extraction; the algorithm is as follows:

1. Calculate the mean based on $x_{i}$ and obtain the expected value $E x=\frac{1}{n} \sum_{i=1}^{n} x_{i}$

2. Calculatethestandarddeviationbased on $x_{i}$ and obtaintheentropy $E n=\sqrt{\frac{1}{n-1} \sum_{i=1}^{n}\left(x_{i}-E x\right)^{2}}$

3. For each couple of $\left(x_{i}, u\left(x_{i}\right)\right)$, calculate $E n_{i}^{\prime}=\sqrt{\frac{-\left(x_{i}-E x\right)^{2}}{2 \ln u\left(x_{i}\right)}}$

Where, the certainty degree $u\left(x_{i}\right)$ corresponding to $x_{i}$ can be determined according to the following formula $u\left(x_{i}\right)=1-\frac{\left|x_{i}-E x\right|}{\max \left(x_{i}\right)-E x}$, which means that the closer the sample is to the expected value, the greater the certainty degree of the sample.

4. Calculate the mean based on $E n_{i}^{\prime}$ and obtain the expected value $E \breve{n}=\frac{1}{n} \sum_{i=1}^{n} E n_{i}$

5. Calculate the standard deviation based on $E n_{i}^{\prime}$ and obtain the hyper entropy

$$
H e=\sqrt{\frac{1}{n-1} \sum_{i=1}^{n}\left(E n_{i}-E \breve{n}\right)^{2}}
$$

Step 3: Identify the concept, which the quantitative data belongs to 
The following algorithm is used to identify the concept, which the given speed or distance data belongs to:

1. Use the X-condition cloud generator to calculate the certainty degree $u_{i}(a)$ that the given value $a$ belongs to a certain qualitative concept $C_{i}$, that is, firstly a normally distributed random number is generated which takes $E n_{i}$ as the expected value and takes $H e_{i}$ as the standard deviation, and then $u_{i}(a)$ is calculated by the following formula: $u_{i}(a)=\exp \left[-\left(a-E x_{i}\right)^{2} / 2\left(E n_{i}^{\prime}\right)^{2}\right]$

2. These certainty degrees are arranged from large to small, and the final certainty degree $u_{i}(a)$ will be selected randomly from the first two large certainty degrees with the probability $p_{i}$, that is, $a$ belongs to the qualitative concept $C_{i}$. Here, $p_{i}=u_{i}(a) / \sum u_{i}(a)$

\subsection{Crossing decision rules extraction}

For a given pedestrian crossing decision table, the decision table reduction can be obtained by attribute reduction and value reduction, and then the rules are obtained from the decision table reduction. Specifically it can be divided into the following five steps:

1. delete the duplicate objects in the table;

2. attribute reduction, that is, delete redundant condition attributes;

3. value reduction, that is, delete the redundant attribute value of each object;

4. obtain the decision table reduction;

5. obtain decision rules.

In this paper, RSES (Rough Set Exploration System) software [16] is used to derive reducts and decision rules from pedestrian crossing decision table. A variety of attribute reduction and value reduction algorithms are provided in the software. Attribute reduction based on discernibility matrix and exhaustive algorithm is selected, which constructs all reducts. Value reduction based on global rules are selected, which scans the training sample object by object and produces rules by matching object against reduct.

\section{STUDY DATA}

Jianshe First Road in Wuhan was chosen as an observation site, where many students from Renjialu Middle School often come out of the school and cross the road to opposite restaurants at noon. As shown in Fig. 1, the two-way road has two lanes on each side. A camera was used to collect the video of pedestrian-vehicle conflict between 12:00 pm and 12:25 pm for four days. On average, 876 adolescent pedestrians and 225 vehicles were recorded each day within the observation phase. The average vehicle speed was $6.1 \mathrm{~m} / \mathrm{s}$. The Adobe Premiere software was used to play the video one by one frame, and one second image can be decomposed into 25 frames, which can be used to obtain time-related data. Direct linear transformation method [17] was used to transform the image coordinates of the object in the video to the corresponding ground coordinates of the object, and then the distance-related data can be obtained based on the ground coordinates of pedestrian and vehicle. The speed can be determined by distance and time. Through the above process, a total of 377 cases of pedestrian-vehicle conflict data were extracted, among which 300 cases were used for decision rule extraction and 77 cases were used for prediction accuracy test. 


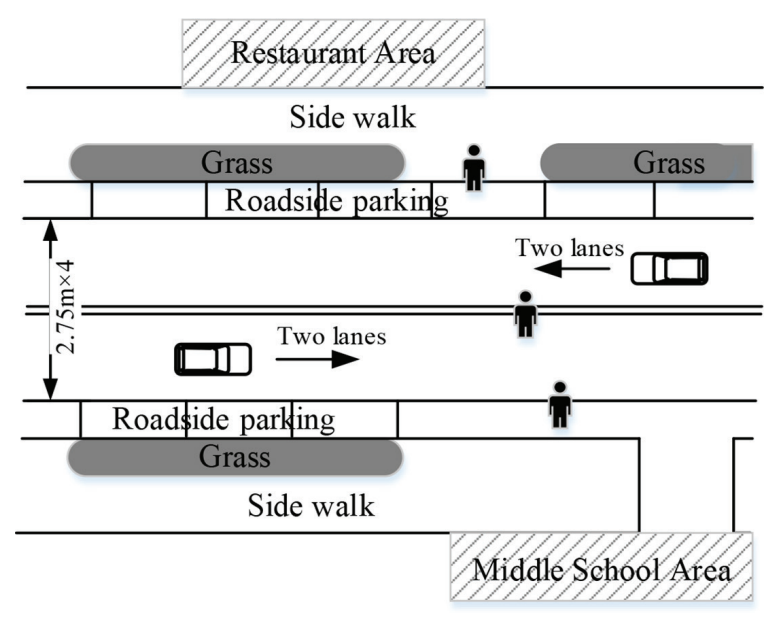

Figure 1: Sketch of roadway for data collection.

\section{RESULTS AND ANALYSIS}

\subsection{Concept classification for influencing factors}

For the vehicle type, two concepts are divided in this paper, that is, passenger car and bus. For group size, three concepts are divided, that is, small ( 1 person), medium ( $2-3$ people) and large (4 people and more). Backward cloud generator mentioned previously was used to determine cloud model parameters of qualitative concepts for some influencing factors such as the vehicle speed and the distance between the vehicle and the pedestrian (Table 1). Figures 2 and 3 depict the cloud model for the concepts of vehicle speed and distance between pedestrian and vehicle, respectively. Clouds from left to right in Fig. 2 represent three concepts of the speed, that is, slow, medium and fast. Clouds from left to right in Fig. 3 represent three concepts of the distance, that is, near, medium and far. The vertical axis in Figs. 2 and 3 represents the certainty degree that a cloud drop belongs to a certain concept.

\subsection{Crossing decision table construction}

Based on the algorithm of concept identification mentioned previously, the data such as the vehicle speed and the distance between the vehicle and the pedestrian are discretized and determined the qualitative concept, so that adolescent pedestrian crossing decision table can

Table 1: Parameters of cloud model for influencing factors.

\begin{tabular}{ccc}
\hline Influencing factors & Qualitative concepts & Parameters $(\mathrm{Ex}, \mathrm{En}, \mathrm{He})$ \\
\hline \multirow{2}{*}{$\begin{array}{c}\text { Distance between vehicle } \\
\text { and pedestrian }(\mathrm{m})\end{array}$} & Near & $(17,6.33,1.54)$ \\
& Medium & $(31.17,5.13,1.05)$ \\
& Far & $(45.75,5.9,1.49)$ \\
\hline \multirow{2}{*}{ Vehicle speed $(\mathrm{m} / \mathrm{s})$} & Slow & $(3.61,0.91,0.2)$ \\
& Medium & $(5.75,1.14,0.24)$ \\
& Fast & $(10.59,1.75,0.48)$ \\
\hline
\end{tabular}




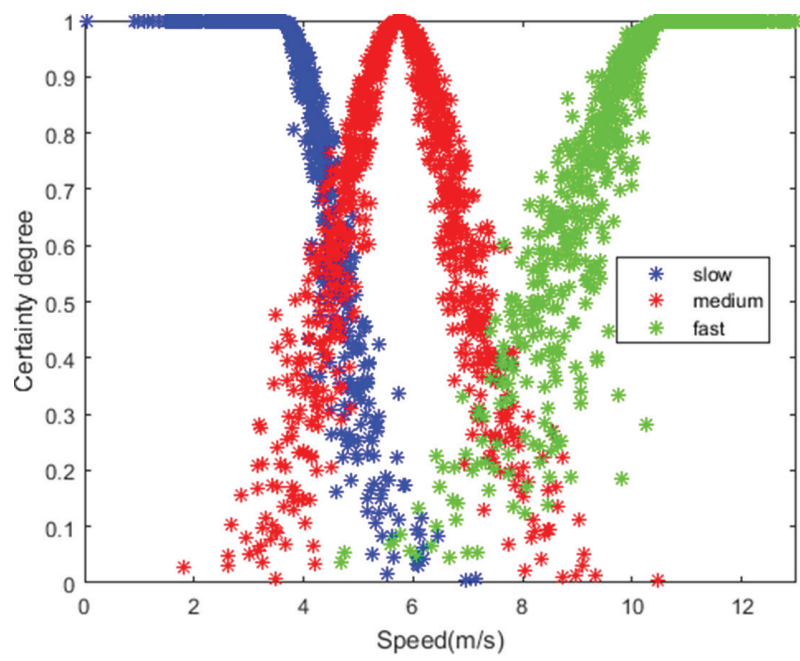

Figure 2: Cloud model for the concept of vehicle speed.

be constructed. In order to facilitate the calculation in rough set theory, the condition attributes of 'distance between pedestrian and vehicle', 'vehicle speed', 'vehicle type' and 'group size' are replaced by letters $a, b, c$ and $d$, respectively; the decision attribute of 'cross or yield' is replaced by the letter $e$; the qualitative concepts of 'near', 'slow' and 'small' are replaced by the value 1 , the qualitative concept of 'medium' is replaced by the value 2 , the qualitative concepts of 'far', 'fast' and 'large' are replaced by the value 3; the qualitative concept of 'cross' is replaced by the value 1, the qualitative concept of 'yield' is replaced by the value 0 . To illustrate the problem, Table 2 gives the crossing decision table.

\subsection{Crossing decision rules extraction}

The RSES software was used to calculate the reducts and generate the rules. The reduct is $\{a, b\}$, which means the vehicle speed and the distance between pedestrian and vehicle

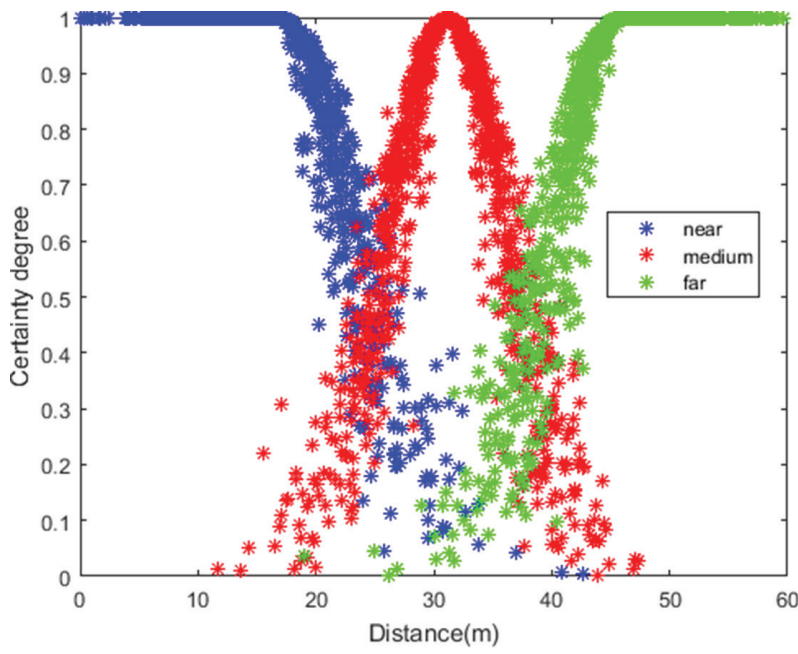

Figure 3: Cloud model for the concept of distance between pedestrian and vehicle. 
have the significant impact on adolescent pedestrian crossing decision. The current results supported previous findings $[7,8]$ that vehicle speed and the distance between pedestrian and vehicle are the important predictors in pedestrian crossing model. Unlike previous findings [2], vehicle type and group size are not significant predictors of pedestrian crossing decision in the current sample. According to the reduction result, nine pedestrian crossing decision rules are extracted including five certainty rules. These rules are as follows.

Rule 1: $a=2 \wedge b=1 \Rightarrow e=1$

Rule 2: $a=2 \wedge b=3 \Rightarrow e=0$

Rule 3: $a=1 \wedge b=2 \Rightarrow e=0$

Rule 4: $a=3 \wedge b=1 \Rightarrow e=1$

Rule 5: $a=1 \wedge b=3 \Rightarrow e=0$

Rule 6: $a=2 \wedge b=2 \Rightarrow e=1(0.125), 0(0.875)$

Rule 7: $a=3 \wedge b=2 \Rightarrow e=1(0.867), 0(0.133)$

Rule 8: $a=1 \wedge b=1 \Rightarrow e=1(0.727), 0(0.273)$

Rule 9: $a=3 \wedge b=3 \Rightarrow e=1(0.111), 0(0.889)$

Some rules are selected to introduce.

(1) certainty rule

$a=2 \wedge b=1 \Rightarrow e=1$, which means that if the distance between pedestrian and vehicle is medium and the vehicle speed is slow, then adolescent pedestrian will choose to cross.

(2) uncertainty rule

$a=2 \wedge b=2 \Rightarrow e=0$, the confidence of the decision rule is 0.875 , which means that if the distance between pedestrian and vehicle is medium and the vehicle speed is medium, then adolescent pedestrian will choose to yield with the probability of $87.5 \%$.

\subsection{Comparison with the other method}

Logistic regression method was applied to compare with the method proposed in this paper. In the SPSS statistical analysis software, the logistic regression model was established by LR (likelihood ratio) forward step method, and the estimated values of the variables (distance between pedestrian and vehicle, vehicle speed) and the constant are $0.159,-1.241$ and 1.583 respectively.

In this paper, two methods were compared by using the accuracy and the area under the receiver operating characteristic (ROC) curve [18] (Fig. 4 and Table 3). The ROC curves illustrate the relationship between the true positive rate (sensitivity) and the false alarm rate (1-specificity) for thresholds from 0 to 1 . According to Fig. 4 and Table 3, it can be seen that

Table 2: Sample of crossing decision table.

\begin{tabular}{cccccc}
\hline Decision sample & $\boldsymbol{a}$ & $\boldsymbol{b}$ & $\boldsymbol{c}$ & $\boldsymbol{d}$ & $\boldsymbol{e}$ \\
\hline 1 & 1 & 1 & 0 & 3 & 1 \\
2 & 2 & 1 & 0 & 2 & 1 \\
3 & 2 & 3 & 1 & 2 & 0 \\
$\ldots$ & $\ldots$ & $\ldots$ & $\ldots$ & $\ldots$ & $\ldots$ \\
\hline
\end{tabular}




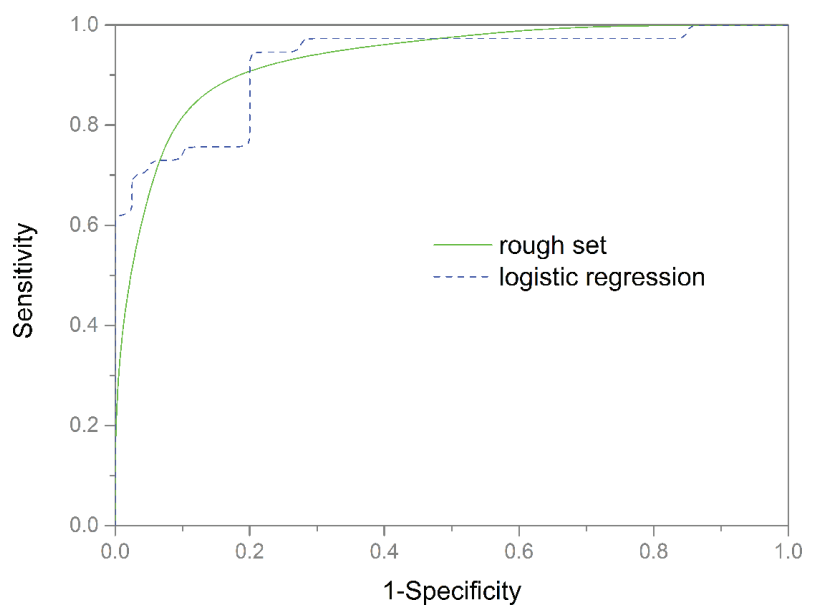

Figure 4: ROC curves for two methods.

Table 3: Comparison of two methods.

\begin{tabular}{ccc}
\hline Methods & Accuracy & Area under the ROC curve \\
\hline Rough set & 0.914 & 0.941 \\
Logistic regression & 0.818 & 0.926 \\
\hline
\end{tabular}

the proposed method has higher index value than logistic regression method, which indicates that the method has high accuracy and can be used to analyze adolescent pedestrian crossing decision behavior.

\section{CONCLUSIONS}

This study considers pedestrian's cognitive fuzziness and randomness in the crossing decision-making process, and uses the cloud model to discretize the continuous data of the influencing factors, and then constructs adolescent pedestrian crossing decision table. Finally, rough set method is used to extract the decision rules for adolescent pedestrian crossing at unmarked roadway. Compared with the existing method, the proposed method not only has better prediction accuracy, but also can extract clear adolescent pedestrian crossing decision rules, which are easy to understand.

The vehicle speed and the distance between pedestrian and vehicle have the significant impact on adolescent pedestrian crossing decision at unmarked roadway. Nine decision rules, including five certainty rules and four uncertainty rules, can describe adolescent pedestrian crossing decision at unmarked roadway.

As a future study, the proposed adolescent pedestrian crossing decision model will be incorporated into the traffic simulation to analyze adolescent pedestrian safety. Another interesting aspect is to expand the sample size and take into account the differences in cognition among different age groups.

\section{ACKNOWLEDGEMENT}

Research for this study was supported by the National Natural Science Foundation of China (No.51208400). 


\section{REFERENCES}

[1] Zhou, R. \& Horrey, W. J., Predicting adolescent pedestrians' behavioral intentions to follow the masses in risky crossing situations. Transportation Research Part F-Traffic Psychology and Behaviour, 13(3), pp. 153-163, 2010. https://doi.org/10.1016/j. trf.2009.12.001

[2] Zhuang, X. \& Wu, C., Pedestrians' crossing behaviors and safety at unmarked roadway in china. Accident Analysis and Prevention, 43(6), pp. 1927-1936, 2011. https://doi. org/10.1016/j.aap.2011.05.005

[3] Xie, S. Q., Wong, S. C., Ng, T. M. \& Lam, W. H. K., Pedestrian crossing behavior at signalized crosswalks. Journal of Transportation Engineering Part A-Systems, 143(8), p. 04017036, 2017. https://doi.org/10.1061/jtepbs.0000055

[4] Sun, D., Ukkusuri, S. V. S. K., Benekohal, R. F. \& Waller, S. T., Modeling of motoristpedestrian interaction at uncontrolled mid-block crosswalks. Presented at the 82nd Annual Meeting of the Transportation Research Board, Washington D.C., 2003. https:// doi.org/10.1177/0361198119850142

[5] Yannis, G., Papadimitriou, E. \& Theofilatos, A., Pedestrian gap acceptance for midblock street crossing. Transportation Planning and Technology, 36(5), pp. 450-462, 2013. https://doi.org/10.1080/03081060.2013.818274

[6] Mamidipalli, S. V., Sisiopiku, V. P., Schroeder, B. J., Elefteriadou, L., Salamati, K. \& Rouphail, N. M., Probit-based pedestrian gap acceptance model for midblock crossing locations. Transportation Research Record, (2519), pp. 128-136, 2015. https://doi. org/10.3141/2519-14

[7] Liu, Y. \& Tung, Y., Risk analysis of pedestrians' road-crossing decisions: effects of age, time gap, time of day, and vehicle speed. Safety Science, 63, pp. 77-82, 2014. https:// doi.org/10.1016/j.ssci.2013.11.002

[8] Pawar, D. S. \& Patil, G. R., Critical gap estimation for pedestrians at uncontrolled midblock crossings on high-speed arterials. Safety Science, 86, pp. 295-303, 2016. https:// doi.org/10.1016/j.ssci.2016.03.011

[9] Papadimitriou, E., Lassarre, S., Yannis, G. \& Tselentis, D. I., Road, traffic, and human factors of pedestrian crossing behavior. Transportation Research Record, (2586), pp. 28-38, 2016. https://doi.org/10.3141/2586-04

[10] Han, X., Yan, Y., Cheng, C., Chen, Y. \& Zhu, Y., Monitoring of oxygen content in the flue gas at a coal-fired power plant using cloud modeling techniques. IEEE Transactions on Instrumentation and Measurement, 63(4), pp. 953-963, 2014. https://doi.org/10.1109/ tim.2013.2287117

[11] Zhao, J., Zhang, K. \& Wan, J., Research of feature selection for text clustering based on cloud model. Journal of Software, 8(12), pp. 3246-3252, 2013. https://doi.org/10.4304/ jsw.8.12.3246-3252

[12] Huang, C., Tseng, T. L., Fan, Y. \& Hsu, C. H., Alternative rule induction methods based on incremental object using rough set theory. Applied Soft Computing, 13(1), pp. 372-389, 2013. https://doi.org/10.1016/j.asoc.2012.08.042

[13] Pai, P. F. \& Lee, F. C., A rough set based model in water quality analysis. Water Resources Management, 24(11), pp. 2405-2418, 2010. https://doi.org/10.1007/s11269009-9558-3

[14] Xu, C. L., Wang, G. Y. \& Zhang, Q. H., A new multi-step backward cloud transformation algorithm based on normal cloud model. Fundamenta Informaticae, 133(1), pp. 55-85, 2014. https://doi.org/10.1007/978-3-642-32115-3_37 
[15] Wang, G. Y., Xu, C. L. \& Li, D. Y., Generic normal cloud model. Information Sciences, 280, pp. 1-15, 2014. https://doi.org/10.1016/j.ins.2014.04.051

[16] Clark, S. D., Characterising and predicting car ownership using rough sets. Transportation Research Part C-Emerging Technologies, 17(4), pp. 381-393, 2009. https://doi.org/10.1016/j.trc.2009.01.006

[17] Yan, W. Y., Shaker, A. \& Easa, S., Potential accuracy of traffic signs' positions extracted from google street view. IEEE Transactions on Intelligent Transportation Systems, 14(2), pp. 1011-1016, 2013. https://doi.org/10.1109/tits.2012.2234119

[18] Xu, C., Wang, W. \& Liu, P., Identifying crash-prone traffic conditions under different weather on freeways. Journal of Safety Research, 46, pp. 135-144, 2013. https://doi. org/10.1016/j.jsr.2013.04.007 